\title{
Aging, fragility and palliative care: challenges of an emerging context
}

\begin{abstract}
Background: It is common, the difficulty among health professionals to recognize the frailty syndrome, multisystemic disease responsible for the physical, cognitive and functional weakness of the elderly. The undervaluation of the general state of the elderly in the clinical routine and the lack of an accurate identification tool result in a late diagnosis. Thus, frail elderly people are subjected to aggressive and nonbeneficial interventions that impair the remaining quality of life due to the suffering caused by iatrogeny.
\end{abstract}

Objective: To study the application of palliative care in the approach to this nonmalignant but life-limiting syndrome, emphasizing the importance of the systematic evaluation of the fragile elderly and the main tools to do so.

Conclusion: Although there is no "gold standard" tool in the evaluation of the elderly's fragility, palliative care promotes, through various analytical methods, the survival of the elderly patient, preserving not only their physical integrity, but also their cognitive, emotional, spiritual values and social insertion.

Keywords: frail elderly, palliative car, sarcopenia, medical oncology, geriatrics
Volume 2 Issue I - 2018

\author{
Mello JLC, Souza DMT, Chacara RAL, Narita \\ RS, Chiminazo LFS \\ Department of Geriatrics, Sapucai Valey University, Brazil
}

Correspondence: Jorge Luiz de Carvalho Mello, Department of Geriatrics, Sapucai Valey University, Pouso Alegre, Minas Gerais, Brazil, Tel (35)3421978,

Email jorgeluis_melo@yahoo.com.br

Received: November 16, 2017| Published: February 07, 2018
Abbreviations: ADL, activities of daily living; ED, emergency department; CCA, comprehensive geriatric assessment

\section{Introduction}

Aging is a continuous process inherent in the human condition. It comprises a whole set of organic, psychic, and social changes of a progressive nature that are influenced by both genetics and the habits and behaviors the individual assumes throughout their life. Aging is marked by the reduction of organic and cognitive functions, a greater predisposition to the development of chronic degenerative diseases, and loss of independence in the activities of daily living (ADLs). ${ }^{1}$ Although there is a decline in physiological functions in this continuous process, which is senescence, aging can occur in an unsuccessful way, with a marked decrease in the quality of life due to one or more illmanaged illnesses that have long time affected the elderly. There is a decline in several systems with energy and functional dysregulation, being a pathological aging, and cannot be associated merely to the years passing by. In this sense, a set of pathological alterations have been studied for the purpose of classification and better understanding and approach in debilitated elderly. This is "fragility".

For many years, the term "fragility" has been used to describe elderly people who are emaciated and have difficulty in getting around, who theoretically are more susceptible to injuries, falls, and morbid outcomes. More recently, Fried et al., ${ }^{2}$ have linked the term to the state of functional decline and vulnerability characterized by weakness and decreased physiological reserve, and for Mcdermid et al. ${ }^{3}$ \& Schoon et al., ${ }^{4}$ frailty syndrome is characterized by reduced physical and cognitive reserves and makes the elderly more vulnerable to adverse events, such as hospitalizations, falls, loss of independence, and death. Still according to Fried et al. ${ }^{2}$ Fragility is closely related to functional disability and comorbidities, with coexistence of the same in $21.5 \%$ of the elderly. The presence of two or more comorbidities is often correlated with the syndrome, and there is a direct causal relationship between them.

For Mello et al., ${ }^{5}$ associations between fragility syndrome and other characteristics can be made. They are advanced age, female gender, black skin color, presence of two or more comorbidities, polypharmacy, functional or cognitive disability, the occurrence of low BMI, obesity in the elderly, depressive symptoms, and self-evaluation poor health. Finally, these associations that Fried et al. ${ }^{2}$ call "fragility phenotype" have statistically significant predictive association with five major outcomes: first hospitalization, first decline, disability in ADLs, worsening of mobility and death. That is, there will certainly be impacts on the quality of life of the elderly, an unsuccessful aging, and as is often the case, in the absence of the possibility of cure, their death.

It is in this scenario that the number of frail elderly patients could benefit from palliative care sooner rather than later in their disease course. It is essential that relief be provided for the suffering caused by the various diseases that over time have taken away the health and lifestyle of both the elderly person and his family, which is worsened by the loss of the elderly functions. Thus, this review aims to relate fragility and palliative care, emphasizing the importance of a systematized evaluation and discuss the feasibility of palliative care in this context.

\section{Discussion}

Recognizing frail patients who are approaching the end of life is complex and often delayed due to clinical uncertainty ${ }^{6}$ and public perception that the end of life is a point in time rather than a process that can take days, weeks, or years. ${ }^{7}$ In fact, the end of life journey can commence years earlier with frailty being one of its salient features. The coexistence of frailty and cognitive impairment and dementia indicates that the dying trajectory has commenced. ${ }^{8}$ 
Still according to Kennedy et al., ${ }^{6}$ delays in diagnosing worsening frailty as a terminal process (i.e. end of life) often lead to aggressive and non-beneficial treatments ${ }^{9}$ that can impair quality of remaining life and increase suffering, foster false hope ${ }^{10}$ and preclude healthy grieving for both patients and their families. According to guidelines, a comprehensive frailty assessment in all older adults is recommended across the continuum of care because it is known that frailty status has a strong association with poor outcomes. ${ }^{11}$ However, it is not routinely measured in some settings, for example in the emergency department (ED), perhaps due to the perceived duration of the assessment in an already busy environment. ${ }^{12}$

Furthermore, reasons for overlooking frailty in the clinical setting is the slowly progressing clinical presentation or attribution of frailty to normal aging process rather than a disease..$^{13}$ Other factors are: the clinicians focus on a specific organ system based model ${ }^{14}$ to which frailty-being a multisystem disease-does not conform; lack of geriatric training by non-geriatric clinicians, ${ }^{15}$ or lack of awareness of frailty tools available to examine older adults. ${ }^{16}$ All of this leads to a delay in recognizing frailty especially in the early stages where targeted interventions to delay or slow-down the progression would be of benefit. ${ }^{17}$

To avoid delay in identification of fragility and all its consequences, it is possible to perceive that some authors are committed in the recognition of the fragility through the systematization of the tools. For example, geriatric oncology studies have trailed the rising population of older adults with cancer. ${ }^{18}$ Among the gaps in knowledge is the need for better risk stratification and treatment selection based on frailty status. ${ }^{19-21}$ For this, it is possible to use mechanisms such as the comprehensive geriatric assessment (CGA) that has been used as a "gold standard" in the oncology literature to identify vulnerable and frail adults. The CGA includes an evaluation of medical, functional, psychological, cognitive, and social health.

It identifies potentially modifiable interventions to maximize independence, social support, cognition, and quality of life while reducing risks for poor outcomes such as delirium, worsening disability, post-operative complications, rehospitalization, or surgical mortality. The CGA identifies not only physical frailty but a wide variety of vulnerabilities. ${ }^{22,23}$ Although there is no consensus in the literature on the criteria for identifying fragility, the model created by Fried et al., is among the most widely used today. For the author, the presence of three or more criteria classifies the elderly as fragile and the presence of one or two classifies them as pre-fragile, understood here as those that present a high risk to develop the fragility syndrome. ${ }^{2}$

The criteria established by Fried ${ }^{2}$ are:

i. Unintentional weight loss of $4.5 \mathrm{~kg}$ or $5 \%$ of body weight in the last year.

ii. Self-rated fatigue, assessed through questions and the depression scale of the Center for Epidemiological Studies.

iii. Reduction of palmar grip strength, measured by manual dynamometer, in the dominant upper limb.

iv. Reduced level of physical activity measured by the weekly energy expenditure in kcal and decrease in walking speed.

The Fried ${ }^{2}$ model is based on sarcopenia and immunological and neuroendocrine changes and focuses on the physical dimension of fragility. Sarcopenia, an important comorbidity which sets in with aging, is characterized by the reduction in the amount and ability of the contractile proteins to generate tension against a resistance. There is loss of muscle mass and its replacement with fat and collagen, altering the body's constitution. With sarcopenia, there is a decrease in both number and size of muscle fibers, resulting in gradual and selective decrease, with a more pronounced involvement of type II muscle fibers and a change in the ratio between fast and slow contraction fibers (type II and I respectively). ${ }^{24}$

In gerontology, stroke velocity is a widely used clinical measure due to the predictive power to identify elderly at risk for falls, hospitalizations, institutionalization, and other undesirable outcomes. Alterations of gait and balance are frequent in the elderly, reflecting in general state of health of the individual, along with cognitive function, which will be determinant for their autonomy. Good correlation between the Fried's fragility score and gait speed was evidenced, which makes this functional test adequate to monitor and assist in the diagnosis of fragility syndrome in the elderly. ${ }^{4}$

Rockwood, ${ }^{25}$ in turn, developed a multidimensional fragility model that contemplated the presence and severity of diseases, the ability to perform activities of daily living, and physical and neurological signs and symptoms from the clinical examination. His model resulted in a clinical fragility scale that classified the elderly in seven strata ranging from the robust elderly to the severely fragile elderly and an index of fragility derived from 70 different clinical deficits, where the sum of the diagnosed items is divided by the total indexes evaluated, providing a measure of proportion. In this model, a value greater than or equal to 0.25 indicates brittleness and between 0.09 and 0.25 classifies the elderly as pre-frail. ${ }^{25}$

There is also another model that studies the fragility syndrome and how to identify it. The fragility defined by the Study of Osteoporotic Fractures Index (SOF) is identified by the presence of two or more criteria:

\section{i. Unintentional weight loss $>$ or $=5 \%$.}

ii. Inability to get up from a chair five times without using arms.

iii. Exhaustion assessed by self-reported fatigue (Identified by the "no" answer to the following question: "Do you feel full of energy?" from the Geriatric Depression Scale). ${ }^{26}$

The systematization of frailty syndrome identification tools favors the initial approach of the elderly, facilitating how and when to intervene to provide a better quality of life for the patient. It is within this scope that palliative care can be extremely beneficial to the patient. Palliative care is an approach that improves the quality of life of patients and their families who are facing problems associated with life-threatening illness. It prevents and relieves suffering through the early identification, correct assessment and treatment of pain and other problems, whether physical, psychosocial or spiritual. Palliative care is usually provided by an interdisciplinary team. ${ }^{2} 7$

Identifying frailty to guide decisions on interventions to slow its progression and early referrals to community and palliative care may be seen as a cost-saving exercise by some. While triaging frail dying people to other services may lead to reduction in acute care costs, in practice, a sense of equity in healthcare and a duty to be honest about poor prognosis are the ethical responses to the escalating problem of high and unrealistic demand for services to prolong life in the absence of benefit. ${ }^{28}$ 
There is evidence of physical training strategies to slow its progression and minimize the complications of frailty such as poor functional outcomes and death. Multidisciplinary teams can play a part in providing active or comfort care based on need. When frailty becomes more advanced, palliative care can help facilitate a good death involving attention to the physical, psychosocial, and spiritual aspects of patient care aligned with patient's informed goals of care. ${ }^{28}$

It is the responsibility of all clinicians both in the community and hospital settings to screen for frailty and associated risk of death among older patients. There needs to be systems of care, especially in the community setting so once frailty is identified, practitioners consult older patients on their personal values, guide the establishment of realistic goals, and refer them to the most appropriate care pathway. ${ }^{28}$ When people are so frail that they cannot withstand interventions that can cause significant injury, such as surgery or chemotherapy, then appropriate end-of-life care should focus on maintaining their highest-order functions..$^{29}$

That is the reason that palliative care, developed initially to provide holistic and timely symptom-based care for patients with noncurable cancer, should also be available and offered to patients with non malignant, life-limiting diseases. ${ }^{30,31}$ Meeting this standard of geriatric care is not easy, due the burden of frailty in this population. Needed are multimodal palliative efforts across the spectrum of settings, from the home to the hospital and nursing home. ${ }^{32}$ To do this, we need to embrace the complexity posed by each person's presentation and view care through the frailty lens. This will give us a common language in which to engage in a conversation with the same goal in mind: optimizing quality of life. ${ }^{29}$ The concept of frailty assessment is therefore essential for the timely delivery of holistic palliative care in geriatrics patients who have progressive and ultimately terminal conditions. ${ }^{29}$

\section{Conclusion}

The intervention in frailty syndrome is of extreme importance due to the severity of the consequences that usually lead the elderly to the loss of their quality of life. For this, it is necessary to recognize it early using the tools of evaluation. Although they are diverse and there isn't a "gold standard", which difficult the application, it is fundamental that these tools are applied, depending on the intended use. Treating the frailty syndrome will likely reduce risk for functional decline and cognitive disability outcomes.

Palliative care is an approach that improves the quality of life of patients and their families who are facing problems associated with life-threatening illness. It helps other issues of the elderly in their survival, preserving not only the physical integrity, but also their social insertion, lack of support, spiritual values. When therapeutic attempts are ineffective, bringing harm to the elderly, palliative care can bring dignity to the end of the patient's life.

\section{Acknowledgements}

None.

\section{Conflict of interest}

The authors do not have interest conflicts.

\section{References}

1. http://www.who.int/mediacentre/factsheets/fs404/en/

2. Fried LP, Tangen CM, Walston J, et al. Frailty in older adults: evidence for a phenotype. J Gerontol A Biol Scie Med. 2001;56(3):146-156.
3. McDermid RC, Stelfox HT, Bagshaw SM. Frailty in the critically ill: a novel concept. Critical Care. 2011;15(1):301.

4. Schoon Y, Bongers K, Van Kempen J, et al. Gait speed as a test for monitoring frailty in community-dwelling older people has the highest diagnostic value compared to step length and chair rise time. Eur J Phys Rehabil Med. 2014;50(6):693-701.

5. Mello AC, Engstrom EM, Alves LC. Fatores sociodemográficos e de saúde associados à fragilidade em idosos: uma revisão sistemática de literatura. Cad Saúde Pública. 2014;30(6):1-25.

6. Kennedy C, Brooks-Young P, Brunton Gray C, et al. Diagnosing dying: an integrative literature review. BMJ Support Palliat Care. 2014;4:263270 .

7. Murray SA, Kendall M, Boyd K, et al. Illness trajectories and palliative care. BMJ. 2005;330(7498):1007-1011.

8. Kulmala J, Nykanen I, Manty M, et al. Association between frailty and dementia: a population-based study. Gerontology. 2014;60(1):16-21.

9. Cardona-Morrell M, Kim J, Turner RM, et al. Non beneficial treatments in hospital at the end of life: a systematic review on extent of the problem. Int J Qual Health Care. 2016;28(4):456-469.

10. Krawczyk M, Gallagher R. Communicating prognostic uncertainty in potential end-of-life contexts: experiences of family members. $B M C$ Palliat Care. 2016;15:59.

11. Ellis G, Marshall T, Ritchie C. Comprehensive geriatric assessment in the emergency department. Clin Interv Aging. 2014;9:2033-2043.

12. BGS. Fit for frailty: consensus best practice guidance for the care of older people living with frailty in community and outpatient settings. British Geriatrics Society, London, UK: Springer; 2014. p. 1-4.

13. Lee L, Heckman G, Molnar FJ. Frailty Identifying elderly patients at high risk of poor outcomes. Can Fam Physician. 2015;61:227-231.

14. Turner G, Clegg A. Best practice guidelines for the management of frailty: a British Geriatrics Society, Age UK and Royal College of General Practitioners report. Age Ageing. 2014;43:744-747.

15. Kennelly SP, Morley D, Coughlan T, et al. Knowledge, skills and attitudes of doctors towards assessing cognition in older patients in the emergency department. Postgrad Med J. 2013;89:137-141.

16. De Lepeleire J, Iliffe S, Mann E, et al. Frailty: an emerging concept for general practice. Br J Gen Pract. 2009;59:e177-e182.

17. Cesari M, Vellas B, Hsu FC, et al. A physical activity intervention to treat the frailty syndrome in older persons - results from the LIFE-P study. $J$ Gerontol Ser A Biol Med Sci. 2015;70(2):216-222.

18. Dale W, Mohile SG, Eldadah BA, et al. Biological, clinical, and psychosocial correlates at the interface of cancer and aging research. $J$ Natl Cancer Inst. 2012;104(8):581-589.

19. Ruiz M, Reske T, Cefalu C, et al. Management of elderly and frail elderly cancer patients: the importance of comprehensive geriatrics assessment and the need for guidelines. Am J Med Sci. 2013;346(1):66-69.

20. Palumbo A, Bringhen S, Ludwig H, et al. Personalized therapy in multiple myeloma according to patient age and vulnerability: a report of the European Myeloma Network (EMN). Blood. 2011;118(17):4519 4529 .

21. Audisio RA, van Leeuwen B. When reporting on older patients with cancer, frailty information is needed. Ann Surg Oncol. 2011;18(1):4-5.

22. van Leeuwen BL, Huisman MG, Audisio RA. Surgery in older cancer patients - recent results and new techniques: worth the investment? Interdiscip Top Gerontol. 2013;38:124-131.

23. Carli F, Brown R, Kennepohl S. Prehabilitation to enhance postoperative recovery for an octogenarian following robotic-assisted hysterectomy with endometrial cancer. Can J Anaesth. 2012.59(8):779-784. 
24. Unicovsky MA. Idoso com Sarcopenia: uma abordagem do cuidado da enfermeira. Rev Bras Enferm. 2004;57(3):298-302.

25. Rockwood K, Song X, MacKnight C, et al. A global clinical measure of fitness and frailty in elderly people. CMAJ. 2005;173(5):489-495.

26. Ensrud KEA, Ewing SK, Cawthon PM, et al. Comparison of Frailty Indexes for the Prediction of Falls, Disability, Fractures and Mortality in Older Men. J Am Geriatr Soc. 2009;57(3):492-498.

27. http://www.who.int/mediacentre/factsheets/fs402/en

28. Cardona-Morrell M, Lewis E, Suman S, et al. Recognising older frail patients near the end of life: What next? Eur J Intern Med. 2017;45:8490 .
29. Rockwood K, Hatalin K. Frailty in older adults: Implications for endof-life care. Clevelend Clinic Journal of Medicine. 2013;80(3):168-174.

30. Mitchell SL, Kiely DK, Hamel MB. Dying with advanced dementia in the nursing home. Arch Intern Med. 2004;164(3):321-326.

31. Birch D, Draper J. A critical literature review exploring the challenges of delivering effective palliative care to older people with dementia. $J$ Clin Nurs. 2008;17(9):1144-1163.

32. Davies E, Higginson IJ, WHO Europe. Better Palliative Care For Older Peolple. Tipolitografia Trabella, Italy: Springer; 2004. p. 1-43. 